\title{
Control of Membrane Surface Roughness and Pattern Wave Length by Changing the Nonsolvent (Water) Influx Rate
}

\author{
K.C. Khulbe ${ }^{a}$, C.Y. Feng ${ }^{a}$, T. Matsuura ${ }^{a, b,{ }^{*}, \text { W.J. Lau }}{ }^{\text {b, }}$, E. Halakoo ${ }^{b}$, R. Jamshidi Gohari ${ }^{b}$ \\ and A.F. Ismail ${ }^{\mathrm{b}}$ \\ ${ }^{a}$ Department of Chemical and Biological Engineering, University of Ottawa, Canada \\ ${ }^{b}$ Advanced Membrane Technology Research Centre (AMTEC), Universiti Teknologi Malaysia, 81310 UTM, \\ Skudai, Johor, Malaysia
}

\begin{abstract}
The control of surface roughness of polyvinylidene fluoride (PVDF), polyethersulfone (PES), polysulfone (PS) and cellulose (CE) membranes was attempted by changing the rate of nonsolvent influx in the phase inversion process. PVDF and CE were chosen to represent membranes of high hydrophobicity and hydrophilicity, respectively, while PES and PS were chosen to represent membranes of intermediate hydrophobicity/-philicity. The concentration of sodium chloride $(\mathrm{NaCl})$ in the aqueous coagulation medium was increased from 0 to $1.9 \mathrm{~mol} / \mathrm{L}$ to decrease the rate of nonsolvent (water) influx in the solvent/nonsolvent exchange process. As well, the effect of polymer concentration and solvent on the surface roughness was investigated with respect to PVDF and PES. It was observed that the membrane surface roughness increased and decreased, respectively, for the hydrophobic PVDF and hydrophilic CE membrane as the rate of nonsolvent influx was decreased. For the PES and PS membranes of intermediate hydrophilic/-philicity, no significant roughness change was observed. The surface roughness tended to increase as the solution viscosity decreased. It was also observed that the pattern wave length of the hydrophobic membrane did not change significantly while that of the hydrophilic membrane increased significantly as the solvent influx rate was reduced. This trend is predictable by considering the shrinking or swelling of the cast polymer solution during the solvent/nonsolvent exchange process.
\end{abstract}

Keywords: Surface roughness, pattern wave length, hydrophobicity/-philicity, AFM, nonsolvent influx rate, membrane.

\section{INTRODUCTION}

It has been known for a long time that surface properties such as surface roughness, pore size, pore size distribution, etc., play an important role in the membrane performance. Research conducted by Bowen et al. for ultrafiltration (UF) membranes has also shown that the surface roughness may correlate with other material characteristics such as the pore size distribution [1]. After the invention of Atomic Force Microscope (AFM) in 1981, a large amount of work has been reported on the measurement of surface roughness by AFM and attempts have been made to correlate the results with the membrane performance, as summarized in the work of Khulbe et al. [2] and Yao et al. [3]. Some of the examples are; Hirose et al. used Scanning Electron Microscope (SEM) and AFM to investigate the relationship between the surface structure of the skin layers of polyamide thin film composite (TFC) reverse osmosis (RO) membranes and their performances [4]. It was found that the RO membrane with the rougher skin layer produces the higher flux, and there exists an approximately linear

*Address correspondence to these authors at the Department of Chemical and Biological Engineering, University of Ottawa, Canada;

Tel: +1613562 5800x6114; E-mail: matsuura@uottawa.ca

Advanced Membrane Technology Research Centre (AMTEC), Universiti Teknologi Malaysia, 81310 UTM, Skudai, Johor, Malaysia; Tel: +6 07 5535926; E-mail: Iwoeijye@utm.my, lau_woeijye@yahoo.com relationship between the surface roughness and the membrane flux. Stamatialis et al. investigated the surface structure of dense and integrally skinned cellulose acetate and cellulose acetate butyrate membranes by using AFM [5]. It was observed that the surface morphology was associated with permeation properties, i.e. the lower the surface roughness the lower the flux and the higher the rejection. Kwak and Ihm used AFM to study the performances of four commercially available aromatic polyamide TFC RO membranes [6]. The results showed that the membrane performance depends primarily on the nature of the thin skin layer in which the roughest membrane has the highest water flux. Madaeni however reported that the rougher the membrane the lower the permeation rate [7].

Despite all the above reports which concluded that the rough surface is a desirable property of the separation membrane, there are several conflicting reports. For example, Vrijenhoek et al. showed a strong correlation between fouling and surface roughness for some RO and nanofiltration (NF) membranes [8]. This was attributed to the enhancement of interactions between colloidal particles with an increase in surface roughness, i.e. colloidal particles preferentially accumulate at the valleys of the rough membrane surface $[8,9]$. As a result, valleys become blocked and fouling becomes more severe for the rough membrane 
surface [8]. On the other hand, Riedl et al. [10], Yan et al. [11] and Li et al. [12] reported that the trend is reversed for organic particles, i.e. the smoother surface enhances the membrane fouling. Thus, the effect of the nano-scale surface roughness on the overall membrane performance is, at best, controversial at present. Moreover, little is known about the effect of surface roughness on the deposition of smaller organic molecules and salt precipitation.

Although a number of reports are found in the literature on the effect of the surface roughness, either desirable or undesirable, on the membrane performance, the reports on the control of the nanoscale roughness by the choice of membrane material and membrane preparation conditions are scarcely found. As early as 1988, Okada et al. studied the pattern formation on the cellulose membrane surface [13]. The patterns were formed in macroscopic micrometer $(\mu \mathrm{m})$ range and were characterized by the amplitude and wavelength. They found that the pattern could be controlled by changing the rate of nonsolvent influx into the cast polymer solution film in the solventnonsolvent exchange that occurred during the coagulation step. The pattern formation was also affected by the cast film thickness. The roughness parameters obtainable in the AFM analysis, on the other hand, are in nanometer $(\mathrm{nm})$ range and may be considered as the microscopic amplitude and wavelength. The question will then arise if the nanoscale roughness is also controllable by applying the same principle, i.e. by changing the rate of nonsolvent influx.

The objective of this work is to examine the effect of the rate of nonsolvent (water) influx into the cast film on the nano-pattern of the membranes made of different polymeric materials. The rate of nonsolvent influx is controlled by changing the sodium chloride $(\mathrm{NaCl})$ concentration in the aqueous solution that is used for the coagulation medium. As well, the effects of the hydrophobicity/-philicity of the membrane polymer, the concentration of polymer and the solvent used to prepare the casting dope are examined.

\section{THEORY}

In this work, integrally skinned asymmetric membranes were fabricated by the wet phase inversion process (WPIP). In WPIP, upon immersing a cast polymer solution film in a nonsolvent coagulation medium (water), solvent (S)/nonsolvent (N) exchange begins to take place. The $\mathrm{N}$ influx depends largely on the properties of the polymeric material of which membrane is prepared, particularly on the hydrophobic/-philic property of the polymer $(\mathrm{P})$. When $\mathrm{P}$ is hydrophobic, $\mathrm{S}$ outflux surpasses $\mathrm{N}$ influx and the membrane tends to shrink. Conversely, when $P$ is hydrophilic, the membrane tends to swell. When $P$ is neither very hydrophilic nor hydrophobic, the membrane tends to neither shrink nor swell. The property of the top skin layer of the integrally skinned asymmetric membrane also depends on the hydrophobicity/-philicity of $\mathrm{P}$. For hydrophobic $\mathrm{P}$, the skin layer is formed fast due to the poor affinity between $\mathrm{P}$ and $\mathrm{N}$ (water) and the narrow miscibility gap on the $\mathrm{P} / \mathrm{S} / \mathrm{N}$ triangular diagram. For hydrophilic $\mathrm{P}$, on the other hand, solidification is delayed due to the wide miscibility gap, and the front of the highly swollen gel layer gradually advances deep into the cast $P$ solution film during the $\mathrm{S} / \mathrm{N}$ exchange.

While the shrinkage/swelling trends are controlled primarily by the hydrophobicity/-philicity of $\mathrm{P}$, the degree of shrinkage/swelling can be fine-tuned by controlling $\mathrm{N}$ influx. It is known that $\mathrm{N}$ (water) influx can be reduced by reducing the activity of water, which can be achieved by dissolving electrolyte solutes into water. The higher the electrolyte concentration, the lower the activity of water and the slower the influx of $\mathrm{N}$ (water) into the cast polymer solution film.

Figure 1a illustrates schematically the change of polymer volume, $v_{p}$, solvent volume, $v_{s}$, nonsolvent volume, $v_{n}$, and the total volume, $v_{\text {tot }}$, of the cast polymer solution film as a function of time, with respect to the hydrophobic $P$. The figure also indicates the time when the solidification of the top skin layer is completed. It should be noted that $\mathrm{v}_{\text {tot }}$ decreases with time (shrinkage) since the rate of $\mathrm{N}$ influx is less than the rate of $S$ outflux. Furthermore, shrinkage is enhanced by supressing the $\mathrm{N}$ influx. It should be noted that $v_{\text {tot }}$ may have a minimum depending on the relative magnitude of $v_{n}$ and $v_{s}$, but it is not shown in Figure 1a.

Figure $\mathbf{1 b}$ illustrates schematically the similar changes of $v_{p}, v_{s}, v_{n}$, and $v_{\text {tot }}$ as a function of time, with respect to hydrophilic $P$. The figure also includes the time required for the gelation. It should be noted that, contrary to hydrophobic polymer, $v_{\text {tot }}$ increases with time (swelling) and the completing of gel layer formation requires far more time than the solidification of the skin layer for the hydrophobic polymer. Furthermore, swelling is reduced by suppressing the $\mathrm{N}$ influx. It is possible that $v_{\text {tot }}$ has a maximum, but it is not shown in Figure 1b. 


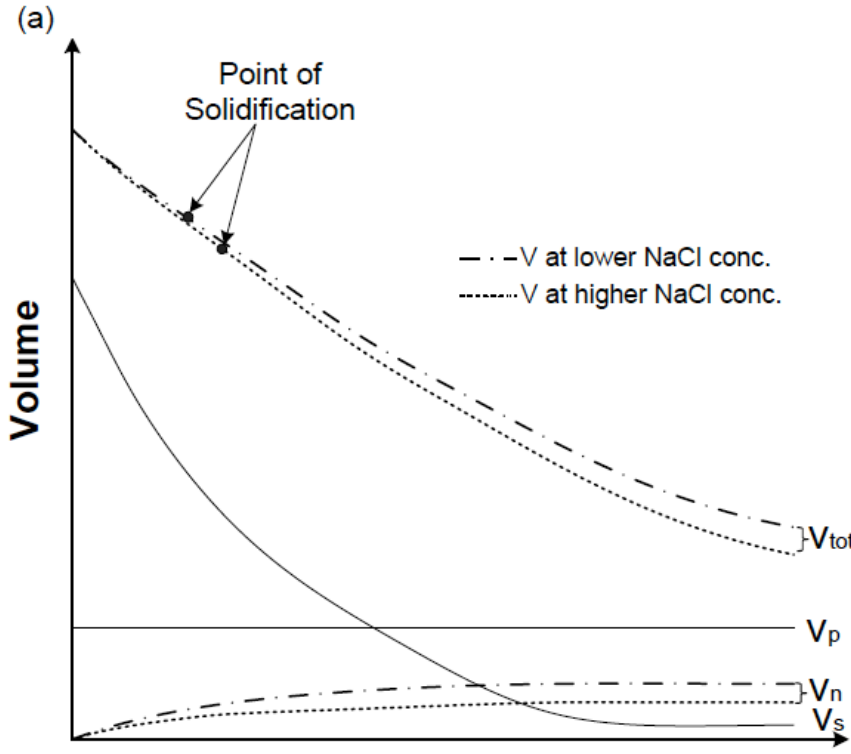

Time

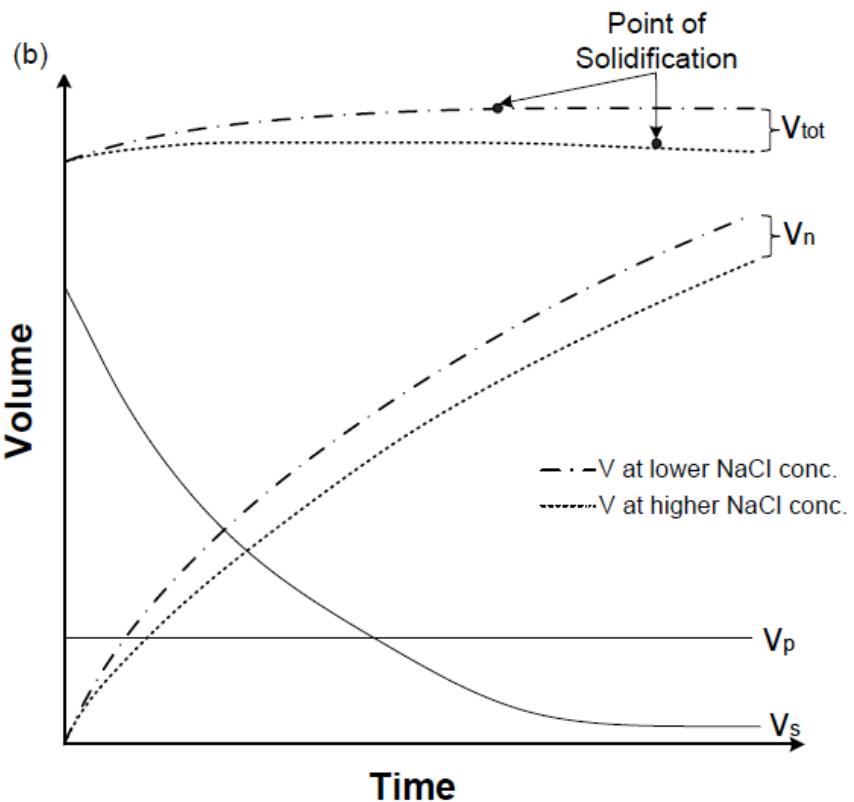

Figure 1: Volume change occurring during phase inversion process, (a) hydrophobic membrane, volume contraction and (b) hydrophilic membrane, volume expansion.

The differences in the $\mathrm{S} / \mathrm{N}$ exchange kinetics are expected to have the following effects on the nanopattern of the membrane surface. In the earlier paper it was argued that, during the S/N exchange in WPIP, the volume expansion of the upper layer of the cast polymer solution results in pattern formation when the expansion of the swollen layer works as compressive force and the latter force is stronger than a critical value [13]. The phenomenon is shown in Figure 2 schematically as the upper surface that is forced to be bent upward. Following the similar argument, the volume contraction will result in pattern formation when the contraction of the shrunk layer works as a decompression force. The phenomenon is shown in Figure 3a schematically as the upper surface that is forced to bend downward. It is also expected that the bending of upper surface, either upward or downward, will be intensified with the decrease in the solution viscosity.

Regarding the effect of the electrolyte concentration in $\mathrm{N}$ (water), it depends on hydrophobicity/-philicity of $P$. In the case of hydrophobic $P$, shrinkage is enhanced by increasing the electrolyte concentration and the deeper downward bending will take place (see Figure 3a), which will increase the amplitude of the membrane surface. On the other hand, in the case of hydrophilic $\mathrm{P}$, swelling is suppressed (Figure $\mathbf{3 b}$ ) and the upward bending becomes less pronounced, which decreases the amplitude of the membrane surface. In the case of $\mathrm{P}$ with intermediate hydrophobicity/-philicity, neither shrinkage nor swelling occurs, resulting in a small amplitude.

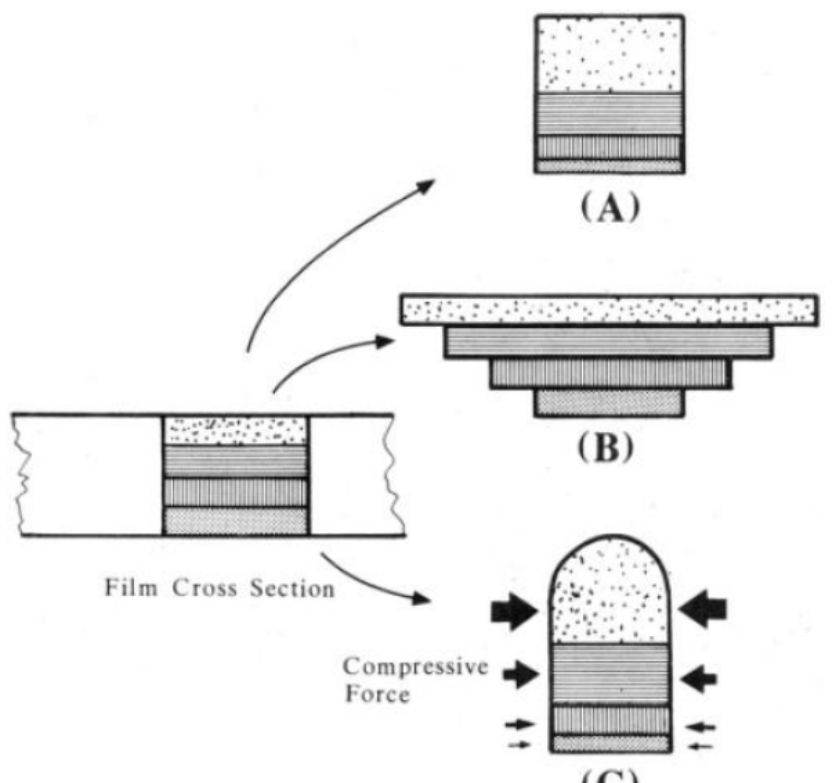

(C)

Figure 2: Surface of hydrophilic membrane bent upward [13].

Regarding the wavelength, it depends on the characteristic length, which in this case is considered to be either the thickness of the skin layer for the hydrophobic membrane or the thickness of the swollen gel layer for the hydrophilic membrane [13]. The skin layer of the hydrophobic membrane is formed fast upon contact of the cast film surface with $\mathrm{N}$ and its thickness does not depend very much on $\mathrm{N}$ influx rate. On the 
(a)
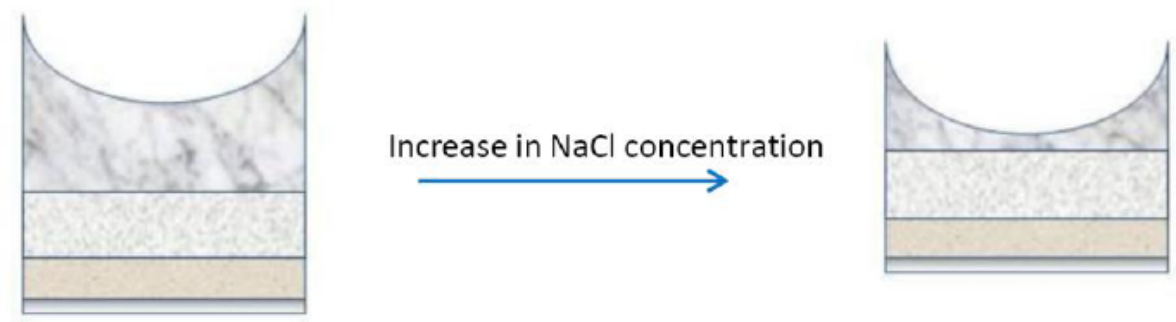

(b)

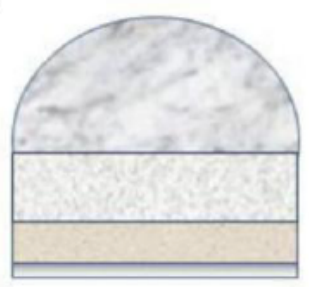

Increase in $\mathrm{NaCl}$ concentration

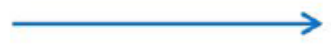

Figure 3: Effect of $\mathrm{NaCl}$ concentration in the coagulation bath on the pattern amplitude, (a) hydrophobic membrane and (b) hydrophilic membrane.

(a)

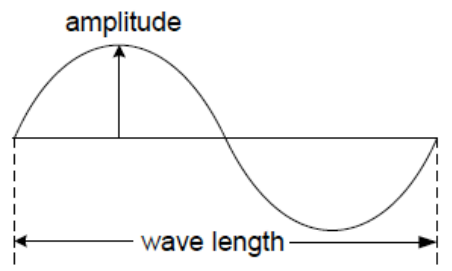

(b)

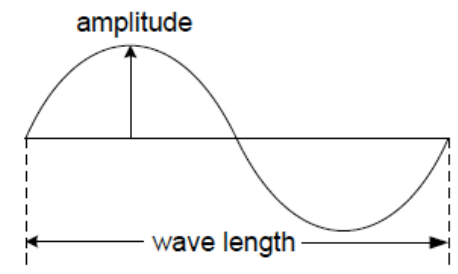

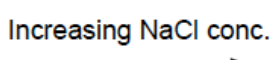

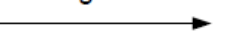

Increasing $\mathrm{NaCl}$ conc.

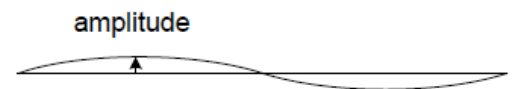

Amplitude decreases

Wave length increases

Figure 4: Effect of $\mathrm{NaCl}$ concentration in the coagulation bath on the amplitude and wave length of the pattern, (a) hydrophobic membrane and (b) hydrophilic membrane.

other hand, the swollen gel of the hydrophilic membrane is formed more slowly and the thickness of the gel layer increases when the rate of $\mathrm{N}$ influx is suppressed due to an increase in delay time. Thus, for the hydrophobic membrane, the wave length changes only little, due to the insignificant change in the characteristic length. On the other hand, the wave length of the hydrophilic membrane will increase significantly, when $\mathrm{N}$ influx is suppressed. Figure $\mathbf{4}$ illustrates schematically the changes in amplitude and wavelength of the pattern with the change in $\mathrm{NaCl}$ concentration in the coagulation medium.

\section{EXPERIMENTAL}

\section{Materials}

Polyvinylidene fluoride (PVDF, Kynar ${ }^{\circledR}$ 761), polyethersulfone (PES, Ultrasan E6020), polysulfone (PS, Udel 3500) and polyvinylpyrrolidone (PVP, $M_{w}=10,000 \mathrm{~g} / \mathrm{mol}$ ) were supplied by Arkema, BASF, Solvay and Sigma-Aldrich, respectively. Cellulose (CE) was supplied from Baker Chemicals in powder form and of chromatography grade. N-methyl-2-pyrrolidone, (NMP) N,N-dimethylacetamide (DMAc) and sodium chloride $(\mathrm{NaCl})$ were purchased from Sigma-Aldrich. 


\section{Membrane Preparation}

\section{PVDF Membrane Preparation}

A required amount of PVDF was added to either NMP or DMAc to prepare the casting dope of polymer concentration, either 14 or $16 \mathrm{wt} \%$. The compositions of the prepared PVDF casting dopes are summarized in Table 1 together with dope viscosity which was measured using a basic viscometer (Cole-Parmer). The mixture was kept stirred at $40^{\circ} \mathrm{C}$ until a uniform solution was obtained. The solution was then cast on a glass plate using a casting blade to a thickness of $30 \mu \mathrm{m}$, before being immersed into coagulation medium immediately at room temperature. Aqueous $\mathrm{NaCl}$ solutions of different concentrations were used for the coagulation medium. After being kept in the coagulation bath for over $12 \mathrm{~h}$, the membrane was separated from the glass plate, washed by distilled water and kept in distilled water. The membrane was dried at room temperature by keeping it on a filter paper before being subjected to AFM observation. Depending on the casting dope composition, these membranes were coded as PVDF-A(B)-dry where A indicates the solvent and $B$ the PVDF concentration, as shown in Table 1.

\section{PS Membrane Preparation}

PS and PVP were added to NMP to prepare the solution of $15 \mathrm{wt} \%$ PS and $7 \mathrm{wt} \%$ PVP. (PVP was added to make the solution casting procedure similar to the conventional UF membrane made of PS). The mixture was kept stirred at $40^{\circ} \mathrm{C}$ until a uniform solution was obtained. The rest of the procedure is the same as for the preparation of PVDF membranes. This sample is called PS-NMP(15)-dry.

\section{PES Membrane Preparation}

Predetermined amounts of PES and PVP were added to either NMP or DMAc to prepare the casting dopes of (18 wt $\%$ PES $/ 9 w t \%$ PVP) or (16 wt $\%$ PES $/ 8$ wt $\%$ PVP). The compositions of the prepared PES dopes are summarized in Table 1. PVP was added to make the solution casting procedure similar to the conventional UF membrane made of PES. The mixture was kept stirred at $40^{\circ} \mathrm{C}$ until a uniform solution was obtained. The rest of the procedure is the same as for the preparation of PVDF membranes. Depending on the dope composition, the membranes were coded as PES-A(B)-dry where $A$ indicates the solvent and $B$ indicates the PES concentration.

\section{CE Membrane Preparation}

(a) CE powder (26.3 g), paraformaldehyde $(25.9 \mathrm{~g})$ potassium hydroxide $(0.14 \mathrm{~g})$ and dimethyl sulfoxide $(243 \mathrm{~g})$ were slurried at room temperature for 30 min in a flask with a stirring device, a thermometer, and a reflux condenser. Then, the flask was placed in a paraffin oil bath. The bath temperature was raised to $125^{\circ} \mathrm{C}$ while the mixture was vigorously stirred and held at $125^{\circ} \mathrm{C}$ for $1 \mathrm{~h}$. Then the mixture was cooled down to $60^{\circ} \mathrm{C}$, and $12.9 \mathrm{~g}$ of paraformaldehyde was added. The temperature was raised again and held at $125^{\circ} \mathrm{C}$ for another hour. The solution was pressure filtered to remove a residual quantity of paraformaldehyde solid and then cooled to room temperature [14]. The membrane preparation was based on the work of Farnand [15] and Uragami et al. [16]. The polymer solution was cast onto a microscope slide made of glass to a thickness of $30 \mu \mathrm{m}$. The cast polymer film together with the glass slide was

Table 1: Casting Dope Composition

\begin{tabular}{|c|c|c|c|c|}
\hline Membrane & $\begin{array}{c}\text { Polymer } \\
\text { (wt.\%) }\end{array}$ & $\begin{array}{c}\text { Additive } \\
\text { (wt.\%) }\end{array}$ & $\begin{array}{c}\text { Solvent } \\
\text { (wt.\%) }\end{array}$ & $\begin{array}{c}\text { Viscosity } \\
\text { (Pa.s) }\end{array}$ \\
\hline PVDF-NMP(16) & PVDF (16) & - & NMP (74) & 3.970 \\
\hline PVDF-NMP(14) & PVDF (14) & - & NMP (76) & 2.050 \\
\hline PVDF-DMAc(16) & PVDF (16) & - & NMP (74) & 1.586 \\
\hline PES-NMP(18) & PES (18) & PVP (9) & NMP (73) & 1.075 \\
\hline PES-NMP(16) & PES (16) & PVP (8) & NMP (76) & 0.700 \\
\hline PES-DMAc(18) & PES (18) & PVP (9) & DMAc (73) & 0.530 \\
\hline PS-NMP(15) & PS (15) & PVP (7) & NMP (78) & - \\
\hline${ }^{\mathrm{a}} \mathrm{CE}$ & \multicolumn{3}{|c|}{ - } & - \\
\hline
\end{tabular}

${ }^{a}$ See the membrane preparation method. 
kept at room temperature for 1 min before being placed in a coagulation medium which was either pure water or aqueous $\mathrm{NaCl}$ solution. The temperature of the coagulation bath was controlled at $20^{\circ} \mathrm{C}$. The glass slide was kept in the coagulation bath for more than $1 \mathrm{~h}$. The membrane was separated from the glass slide and dried at room temperature by keeping the membrane on a filter paper before being subjected to AFM observation. This sample is called CE-dry.

(b) In another experiment, wet CE membrane was prepared by keeping the membrane intact on the glass slide without undergoing drying process. Prior to AFM observation, the excess water on the wet membrane surface was blotted by a tissue paper. A small piece of CE membrane together with the glass slide was placed on the magnetic disk and the surface of the membrane was subjected to AFM observation. The observation was done within $30 \mathrm{~min}$ from the moment when the membrane (together with the glass plate) was taken from the coagulation bath. This sample is called CE-wet.

(c) After AFM analysis, the CE-wet membrane from (b) was dried naturally on the glass plate for $24 \mathrm{~h}$ before being subjected to AFM observation again. This membrane sample is called CE-wetdry.

\section{AFM Characterization}

The 2D and 3D images of the top surface were obtained using tapping mode atomic microscopy (TM AFM) on a Nanoscope III equipped with 1553D scanner, Digital Instruments, Santa Barbara, CA, USA. Several important membrane surface properties were measured such as the roughness parameter, $\mathrm{Ra}$, the nodule size and the pore size. The roughness parameter was used as the amplitude of the pattern. The nodule size and the pore size were considered as the size of the mountain and that of the valley, respectively, as described later, and their sum was used as the wave length of the pattern.

On the other hand, the mountain width $\left(\mathrm{W}_{\mathrm{m}}\right)$ and the valley width $\left(\mathrm{W}_{\mathrm{v}}\right)$ obtained from the AFM line profile (see Figure 5) could be used to calculate two important parameters as follows.

$\mathrm{W}_{\mathrm{wl}}=\mathrm{W}_{\mathrm{m}}+\mathrm{W}_{\mathrm{v}}$

$\Delta \mathrm{W}=\left(\mathrm{W}_{\mathrm{m}}-\mathrm{W}_{\mathrm{v}}\right)$

where $W_{w l}$ corresponds to the wave length of the pattern and $\Delta \mathrm{W}$ represents the position of the horizontal line shown schematically in Figure 4. If $\Delta W$ is positive, the horizontal line is closer to the bottom of the valley. Conversely, if $\Delta \mathrm{W}$ is negative it is closer to the top of the mountain.

\section{RESULTS AND DISCUSSION}

Table 2 summarizes all the experimental roughness data obtained from the above experiments. It should be noted that the roughness parameter is considered as representing the amplitude of the pattern.

Comparing the roughness parameters of PVDF$\operatorname{NMP}(16)$ obtained at two different laboratories independently, the trend (increase in roughness parameter with an increase in the $\mathrm{NaCl}$ concentration in the coagulation bath) was reproduced even though some differences in the magnitude of the roughness parameters were observed. The above trend is expected for the membrane made of hydrophobic $P$ (see Figure 3a). The results are in consistent with the high contact angle value $\left(89^{\circ}\right)$ of PVDF membrane which is the highest among the polymers tested in this work.

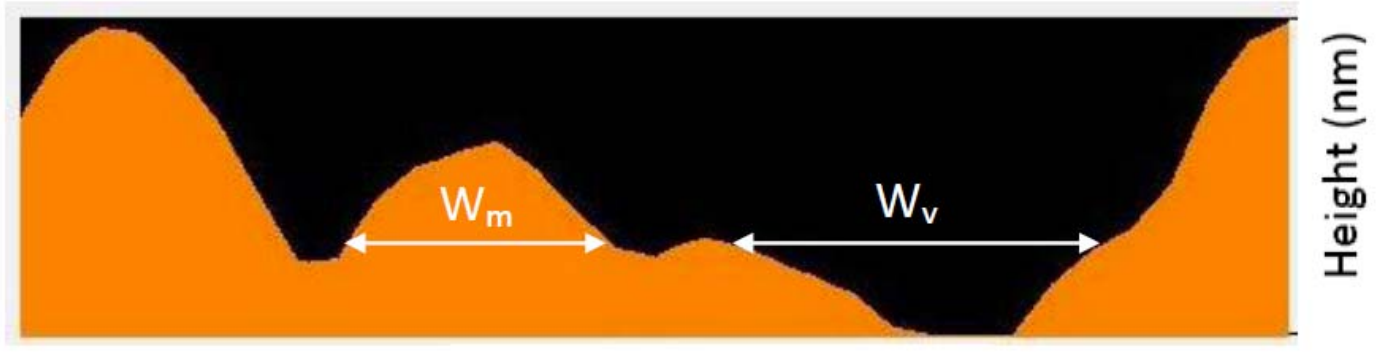

Horizontal distance $(\mathrm{nm})$

Figure 5: Measurement of $W_{m}$ and $W_{v}$ from line profile. 
Table 2: Surface Roughness of Membranes Made of Different Polymeric Materials at Different $\mathrm{NaCl}$ Concentration in the Coagulation bath

\begin{tabular}{|c|c|c|c|c|}
\hline \multirow{3}{*}{$\begin{array}{c}\text { Membrane post } \\
\text { treatment }\end{array}$} & \multirow{3}{*}{ Membrane } & \multicolumn{3}{|c|}{$\mathrm{NaCl}$ concentration in coagulation bath (mol/L) } \\
\hline & & 0 & 0.9 & 1.9 \\
\hline & & \multicolumn{3}{|c|}{ Roughness parameter, $R_{a}(\mathrm{~nm})$} \\
\hline \multirow{9}{*}{$\begin{array}{l}\text { Membrane dried between } \\
\text { filter paper }\end{array}$} & aDYRC NMP(10) dru & 3.48 & 5.10 & 6.56 \\
\hline & rvet-tivir (10)-ciry & 3.83 & 7.50 & 8.33 \\
\hline & PVDF-NMP(14)-dry & 6.19 & 8.11 & 9.20 \\
\hline & PVDF-DMAc(16)-dry & 7.36 & 9.64 & 11.22 \\
\hline & PES-NMP(18)-dry & 2.43 & 2.07 & 1.60 \\
\hline & PES-NMP(16)-dry & 2.72 & 2.50 & 2.85 \\
\hline & PES-DMAc(18)-dry & 5.11 & 4.47 & 4.60 \\
\hline & PS-NMP(15)-dry & 1.88 & 1.82 & 1.47 \\
\hline & CE-dry & 4.6 & 3.1 & 4.6 \\
\hline \multirow{2}{*}{$\begin{array}{l}\text { Membranes kept wet on } \\
\text { glass plate }\end{array}$} & CE-wet & 5.9 & 3.0 & 2.6 \\
\hline & CE-wet-dry & 6.4 & 2.9 & 2.7 \\
\hline
\end{tabular}

${ }^{\mathrm{a}} R_{a}$ data were produced in two independent laboratories.

The roughness parameter increases from PVDF$\operatorname{NMP}(16)$ to PVDF-NMP(14) and further to PVDF$\operatorname{DMAc}(16)$. Referring to Table 1, it is also found that the increase in roughness parameter parallels to the decrease in viscosity, as expected. Interestingly, the trend (increase in roughness parameter with an increase in the $\mathrm{NaCl}$ concentration in the coagulation bath) was maintained regardless of the dope viscosity. Figure 6 presents the 3D AFM images of the PVDF-
$\operatorname{NMP}(16)$ and PVDF-DMAc(16) membranes made of different $\mathrm{NaCl}$ concentration in the coagulation bath.

As for the most hydrophilic CE membrane, with a contact angle of $30^{\circ}$, the roughness parameter decreases with increasing $\mathrm{NaCl}$ concentration from 0 to $0.9 \mathrm{~mol} / \mathrm{L}$, as expected by the theory (see Figure $\mathbf{3 b}$ ). However, with further increase in the $\mathrm{NaCl}$ concentration to $1.9 \mathrm{~mol} / \mathrm{L}$, the roughness of $\mathrm{CE}$
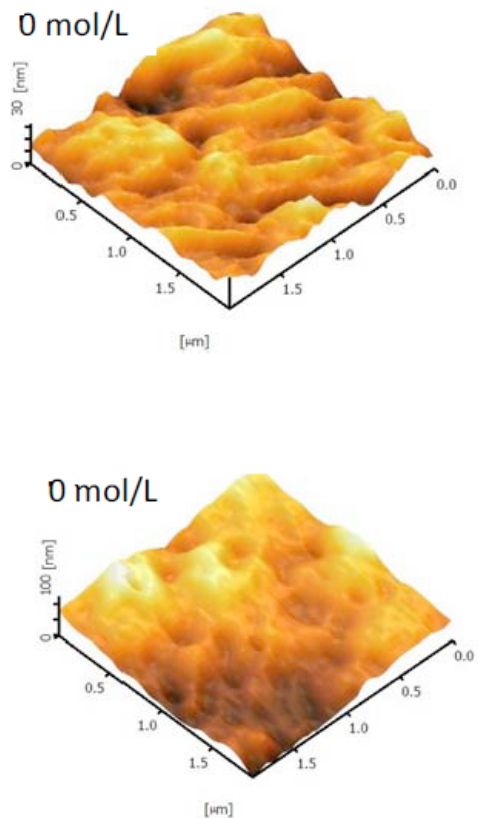
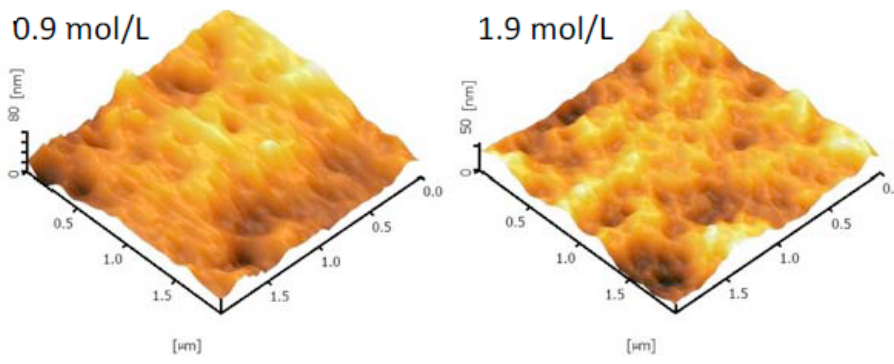

(a)

$0.9 \mathrm{~mol} / \mathrm{L}$

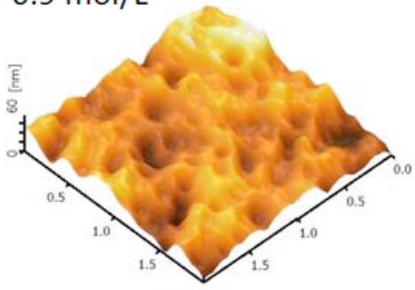

(b)

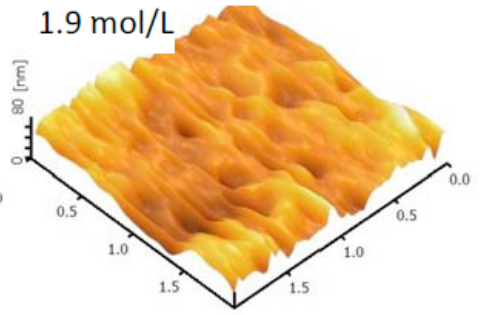

[ $\mu \mathrm{m}]$

Figure 6: 3D AFM images of PVDF membranes made at different $\mathrm{NaCl}$ concentration in the coagulation bath, (a) PVDF$\operatorname{NMP}(16)$-dry and (b) PVDF-DMAc(16)-dry. 
membrane tends to increase, which is contrary to the expectation.

One of the possible reasons for this unexpected result may be due to the membrane post treatment process, i.e. all membranes were replaced from the glass plate onto a filter paper and air dried at the ambient temperature before the AFM observation. In this process, the highly swollen CE membrane shrank considerably, while the size of the little swollen PVDF, PES and PS membranes remained practically unchanged. The shrinkage of the CE membrane should have significant effect on the measured surface roughness. Therefore, in the next experiment the CE membrane was kept wet on the glass plate without drying, to prevent the shrinkage (CE-wet). As can be seen from Table 2 for CE-wet membrane, the membrane roughness continues to decrease with an increase in $\mathrm{NaCl}$ concentration from 0 to $1.9 \mathrm{~mol} / \mathrm{L}$, as expected for the hydrophilic membrane. Interestingly, the roughness of CE-wet-dry membrane remained almost the same even after the membrane was kept on the glass surface for $24 \mathrm{~h}$.

In comparison to PVDF and CE membranes, the roughness parameters of PES-NMP(18)-dry and PSNMP(15)-dry membranes were much lower and decreased slightly as the $\mathrm{NaCl}$ concentration in the coagulation bath increased, which was expected theoretically for the membranes made of $\mathrm{P}$ of intermediate hydrophobicity/-philicity values. The contact angles of PES and PS membranes are $66^{\circ}$ and $70.5^{\circ}$, respectively compared to $89^{\circ}$ displayed by hydrophobic PVDF membrane and $30^{\circ}$ by hydrophilic $\mathrm{CE}$ membrane. One might argue that the addition of PVP in the casting solution may have increased the hydrophilicity of PES and PS membranes. To justify the use of PS/PVP and PES/PVP mixtures instead of pure PS and PES polymer, the solubility parameter was calculated for PVDF, PS/PVP (w/w=15/7), PES/PVP $(\mathrm{w} / \mathrm{w}=2 / 1)$ and $\mathrm{CE}$ membranes. For $\mathrm{PS} / \mathrm{PVP}$ $(w / w=15 / 7)$ and PES/PVP $(w / w=2 / 1)$ mixture, the additivity rule was applied in terms of weight fraction of PVP, using the individual solubility parameter values for PS $\left(11.9 \mathrm{cal} / \mathrm{cm}^{3}\right)$, PES $\left(13.9 \mathrm{cal} / \mathrm{cm}^{3}\right)$ and PVP $\left(15.0 \mathrm{cal} / \mathrm{cm}^{3}\right)$. The increasing order in the solubility parameter was found to be PVDF $\left(7.5 \mathrm{cal} / \mathrm{cm}^{3}\right)<$ PS/PVP $(\mathrm{w} / \mathrm{w}=15 / 7) \quad\left(12.9 \mathrm{cal} / \mathrm{cm}^{3}\right)<\mathrm{PES} / \mathrm{PVP}$ $(\mathrm{w} / \mathrm{w}=2 / 1) \quad\left(14.3 \mathrm{cal} / \mathrm{cm}^{3}\right)<C E\left(24.1 \mathrm{cal} / \mathrm{cm}^{3}\right)$. Therefore, it can be concluded that PVDF is the most hydrophobic, CE is the most hydrophilic and PS/PVP and PES/PVP are of intermediate hydrophobicity/philicity. With respect to PES membranes, the roughness parameter increased in the order PESNMP(18)-dry < PES-NMP(16)-dry < PES-DMAc(18)dry due to the decrease of dope viscosity.

Table 3 summarizes the average values of $W_{m}$ and $\mathrm{W}_{v}$ obtained from the AFM line profile. Using the data in Table 3 and the results of $\mathrm{W}_{\mathrm{wI}}$ and $\Delta \mathrm{W}$ are calculated and tabulated in Table 4. As can be seen, only the data for 0 and $1.9 \mathrm{~mol} / \mathrm{L}$ (the highest $\mathrm{NaCl}$ concentration) are shown to demonstrate the largest changes that occurred with the change of the $\mathrm{NaCl}$ concentration.

Comparing the data of the most hydrophobic (PVDF) and the most hydrophilic (CE) membrane, the following two distinct features could be found on these two membranes.

1) With an increase in $\mathrm{NaCl}$ concentration in the coagulation bath, wave length changes only little for the hydrophobic membrane $(7.9 \%)$ while the change is very significant for the hydrophilic membrane (137\%).

2) $\Delta \mathrm{W}$ is positive for the hydrophobic membrane while it is negative for the hydrophilic membrane for both 0 and $1.9 \mathrm{~mol} / \mathrm{L} \mathrm{NaCl}$ concentrations without exception.

Table 3: Average Width of Mountain, $W_{m}$, and Valley, $W_{v}$, Obtained from the AFM Line Profile for Different Types of Membranes

\begin{tabular}{|c|c|c|c|c|}
\hline & \multicolumn{4}{|c|}{$\mathrm{NaCl}$ concentration in the coagulation bath $(\mathrm{mol} / \mathrm{L})$} \\
\hline & & & & \\
\hline \multirow[t]{2}{*}{ Membrane } & \multicolumn{4}{|c|}{ Width (nm) } \\
\hline & Mountain $\left(\mathrm{W}_{\mathrm{m}}\right)$ & Valley $\left(\mathrm{W}_{\mathrm{v}}\right)$ & Mountain $\left(\mathrm{W}_{\mathrm{m}}\right)$ & Valley $\left(W_{v}\right)$ \\
\hline PVDF-NMP(16)-dry & 30.0 & 21.9 & 29.5 & 26.5 \\
\hline PES-NMP(18)-dry & 12.9 & 11.3 & 17.3 & 15.0 \\
\hline PS-NMP(15)-dry & 31.1 & 19.7 & 25.9 & 26.4 \\
\hline CE-wet & 15.9 & 16.4 & 34.6 & 41.8 \\
\hline
\end{tabular}


Table 4: $W_{\mathrm{wl}}$, and $\Delta \mathrm{W}$ of Membranes Calculated by Equations (1) and (2), Respectively

\begin{tabular}{|c|c|c|c|c|}
\hline \multirow{3}{*}{ Membrane } & \multicolumn{4}{|c|}{$\mathrm{NaCl}$ concentration in the coagulation bath (mol/L) } \\
\hline & \multicolumn{2}{|c|}{0} & \multicolumn{2}{|c|}{1.9} \\
\hline & $\begin{array}{c}W_{\mathrm{wl}} \\
(\mathrm{nm})\end{array}$ & $\begin{array}{c}\Delta \mathrm{W} \\
(\mathrm{nm})\end{array}$ & $\begin{array}{c}W_{w l} \\
(n m)\end{array}$ & $\begin{array}{c}\Delta \mathrm{W} \\
(\mathrm{nm})\end{array}$ \\
\hline PVDF-NMP(16)-dry & 51.9 & 8.12 & 56.0 & 3.00 \\
\hline PES-NMP(18)-dry & 24.2 & 1.72 & 32.3 & 2.30 \\
\hline PS-NMP(15)-dry & 50.8 & 11.4 & 52.3 & -0.50 \\
\hline CE -wet & 32.3 & -0.50 & 76.4 & -7.20 \\
\hline
\end{tabular}

The PES and PS membranes lie in between without showing any features as distinct as the PVDF and CE membranes (A high $\Delta \mathrm{W}$ value of PS-dry at $1.9 \mathrm{~mol} / \mathrm{L}$ is a notable exception). The first feature about the wavelength is exactly what we have expected from the theoretical consideration (see Figure 4). The large wavelength values of the PVDF membrane are probably due to the spinodal decomposition that occurred at the top skin layer of the PVDF membrane. The second feature demonstrates that the valley becomes dominant by bringing the horizontal line closer to the bottom of the valley for the hydrophobic membrane where shrinkage occurs and the mountain becomes dominant by bringing the horizontal line closer to the top of the mountain for the hydrophilic membrane when swelling occurs.

\section{CONCLUSION}

From the above experimental results the following conclusions can be drawn:

1) The fundamental principles involved in the macroscopic pattern formation seem applicable to the formation of nanoscale roughness on the polymeric membrane surface.

2) The membrane surface roughness can be practically controlled by changing the nonsolvent influx during phase inversion process.

3) For hydrophobic PVDF membrane, the roughness increases as the rate of nonsolvent influx decreases. Hydrophilic CE membrane, however, shows the opposite trend.

4) For PES and PS membranes of intermediate hydrophobicity/-philicity, the roughness does not change very much by varying the rate of nonsolvent influx.
5) For hydrophobic membrane, the wave length of the pattern does not change significantly as the rate of nonsolvent influx decreases.

6) For hydrophilic membrane, the wave length of the pattern increases significantly as the rate of nonsolvent influx decreases.

\section{REFERENCES}

[1] Bowen WR, Doneva TA. Atomic force microscopy characterization on ultrafiltration membranes: correspondence between surface pore dimensions and molecular weight cut-off. Surf Interface Anal 2000; 29(8): 544-549.

http://dx.doi.org/10.1002/1096-9918(200008)29:8<544::AIDSIA901>3.0.CO;2-4

[2] Khulbe KC, Feng CY, Matsuura T. Synthetic polymeric membranes: characterization by atomic force microscopy; Springer-Verlag, Berlin and Heidelberg 2008.

[3] Yao Y, Guo S, Zhang Y. Surface properties of reverse osmosis membrane. J Appl Polym Sci 2007; 105: 1261-1266. http://dx.doi.org/10.1002/app.25656

[4] Hirose $\mathrm{M}$, Ito $\mathrm{H}$, Kamiyama $\mathrm{Y}$. Effect of skin layer surface structures on the flux behaviour of RO membranes. J Membr Sci 1996; 121: 209-215. http://dx.doi.org/10.1016/S0376-7388(96)00181-0

[5] Stamatialis DF, Dias CR, Norberta de Pinho M. Atomic force microscopy of dense and asymmetric cellulose-based membranes. J Membr Sci 1999; 160: 235-242. http://dx.doi.org/10.1016/S0376-7388(99)00089-7

[6] Kwak SY, Ihm DW. Use of atomic force microscopy and solid-state NMR spectroscopy to characterize structureproperty-performance correlation in high-flux reverse osmosis (RO) membranes. J Membr Sci 1999; 158: 143-153. http://dx.doi.org/10.1016/S0376-7388(99)00039-3

[7] Madaeni SS. The effect of surface characteristics on RO membrane performance. Desalination 2001; 139: 371. http://dx.doi.org/10.1016/S0011-9164(01)00334-4

[8] Vrijenhoek EM, Hong S, Elimelech M. Influence of membrane surface properties on initial rate of colloidal fouling of reverse osmosis and nanofiltration membranes. J Membr Sci 2001; 188: $115-128$ http://dx.doi.org/10.1016/S0376-7388(01)00376-3

[9] Jamshidi Gohari R, Lau WJ, Matsuura T, Ismail AF. Effect of surface pattern formation on membrane fouling and its control in phase inversion process. J Membr Sci 2013; 446: 326-331.

http://dx.doi.org/10.1016/j.memsci.2013.06.056 
[10] Riedl K, Girard B, Lencki RW. Influence of membrane structure on fouling layer morphology during apple juice clarification. J Membr Sci 1998; 139: 155-166. http://dx.doi.org/10.1016/S0376-7388(97)00239-1

[11] Yan L, Li YS, Xiang CB, Xianda S. Effect of nano-sized $\mathrm{TiO}_{2}$ particle addition on PVDF ultrafiltration membrane performance. J Membr Sci 2006; 276: 162-167. http://dx.doi.org/10.1016/j.memsci.2005.09.044

[12] Li YS, Yan L, Xiang CB, Hong LJ. Treatment of oily wastewater by organic-inorganic composite tubular ultrafiltration (UF) membranes. Desalination 2006; 196: 7683.

http://dx.doi.org/10.1016/j.desal.2005.11.021

[13] Okada T, Matsuura T. Pattern formation on the surface of cellulose membranes prepared by phase inversion technique. Ind Eng Chem Res 1988; 27: 1335-1338. http://dx.doi.org/10.1021/ie00079a040
[14] Johnson DC, Nicholson MD, Haigh FC. Dimethyl sulfoxide/paraformaldehyde: a nondegrading solvent for cellulose. Appl Polym Symp 1976; 28: 931-943.

[15] Farnand BA. A Study of Reverse Osmosis Separation Involving Nonaqueous Solutions, PhD Thesis, University of Ottawa, Ottawa 1983; p. 184.

[16] Uragami T, Sugitani Y, Sugihara M. Studies on syntheses and permeabilities of special polymer membranes: 33 . Permeation behaviour of aqueous alcohol solution through cellulose membranes. Polym 1982; 23: 192-196. http://dx.doi.org/10.1016/0032-3861(82)90299-3 